Original Research Paper

\title{
Human Iris Recognition Based on Hybrid Technique
}

\author{
${ }^{1}$ Asaad Noori Hashim and ${ }^{2}$ Bushraa Mahdi Al-Hashimi \\ ${ }^{1}$ Department of Computer Science, Faculty of Computer Science and Mathematics, University of Kufa, Iraq \\ ${ }^{2}$ Department of Computer Science, Faculty of Education, University of Kufa, Iraq
}

\section{Article history}

Received: 25-08-2019

Revised: 18-11-2019

Accepted: 03-12-2019

Corresponding Author:

Asaad Noori Hashim

Department of Computer

Science, Faculty of Computer

Science and Mathematics,

University of Kufa, Iraq

Email: Asaad.alshareefi@uokufa.edu.iq

\begin{abstract}
Iris recognition is a biometric technique that uses iris pattern information to detect person identification. Initially, the system find out the boundary of the pupil and iris. Then, Circular Hough transform used to find out the center of both pupil and iris in order to crop iris part from the eye image. After that, Daugman's Rubber Sheet model utilized for performing the normalizing step. Then, features extracted based on Legendre moment and Local Quantized. Several orders value with many region of iris have been used to get best value, which satisfied the highest recognition rate. Matching was performed by City Block Distance. The simulation was carried out using samples from CASIA.v4-Interval database, the main tool for programming is MATLAB.
\end{abstract}

Keywords: Iris Recognition, Biometric, Feature Extraction, Legendre, Local Quantized Pattern (LQP)

\section{Introduction}

In this automated world, there is a rapid development in modern science and technology and a widespread use of computers and electronic devices along with a growing world population. The main problem, however, is security in different aspects that necessitates the need for a very precise and reliable authentication technology. Authentication plays a fundamental role, as it is first line of defense against intruders. Traditional systems should, therefore be replaced by accurate, convenient and effective alternatives. In addition, governments and private sectors are increasingly encouraging the use of biometric systems.

The three basic types of authentication system are something already known such as a passwords, something you got such as a card or token and something you such as biometric measures.

Any physiological or behavioral attribute is biometric if satisfies the following criteria:

- Universality all humans have it

- Distinctiveness: Be as different as each individual

- Invariance: not change over time

- Collectability: Easily collectible in terms of acquisition, Digitization and feature extraction from the population

- Performance: The availability of data collection and guarantee to achieve high accuracy

- Acceptability: The readiness of the population to present that attribute to the recognition system
Biometric identifiers are categorized either as physiological or behavioral. The physiological type is specifically related to the shape of the body (e.g., fingerprint, palm veins, face recognition, DNA, palm print, hand geometry, iris recognition, retina and odor/scent). The behavioral category is related to behavioral nature of human beings (e.g., rhythm, gait and voice). These biomedical features are unique, remain constant for each person and can be used to identify individuals owning to the difficulty to replicate and reuse by someone other than a biometric owner.

Automated identification systems based on iris recognition is often known to be the most reliable of all biometric methods. The probability of finding two persons with identical iris pattern is almost zero. Iris has several advantages. First, it is characterized by a unique texture pattern, it has a very rich and complex random form that includes the unique features of each individual and is not affected by genetic factors but is only affected by the primary environment of the fetal. It is remarkable that even twins have a different texture of the iris and even in the same person the left eye pattern is different from the right eye. Second, the iris begins to form during the third month of pregnancy. The iris pattern is largely shaped by the age of three years and is almost constant throughout the life in the absence of external damages. Third, unlike other biomedical properties, the iris is protected from external environment by corneal unless there is an eye disease. 


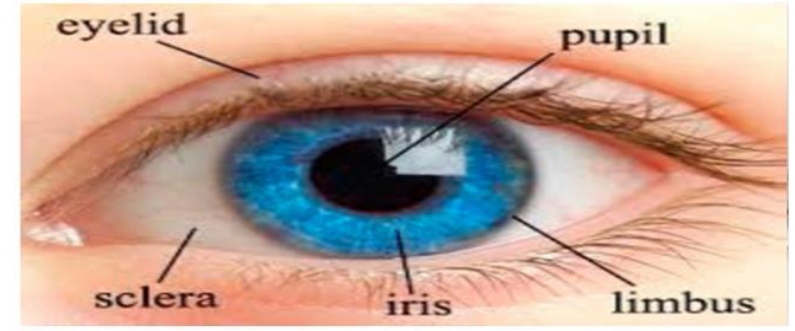

Fig. 1: Human iris

\section{The Human Iris}

Iris is the colored circular region of the eye. It is close to its center, the pupil which is a circular hole. Iris consists of the sphincter and the dilator muscles, which adjust the space of the pupil and therefore, control the amount of light entering through the pupil. The average diameter of the iris is $12 \mathrm{~mm}$. The differentiation is shaped by fibrous and cellular structures such as ligaments, grooves, cysts, rings, frills, crowns, eyelashes, sometimes moles, freckles, components of human eyes have been explained in Fig. 1.

\section{Biometric History}

The idea of using personal identity patterns is proposed in 1936 by ophthalmologist Frank Burch. By 1980, the idea appeared in the James Bond's films, but it was still science imagination and guesswork. In 1987, two ophthalmologists Aram Safir and Leonard Flom acquitted this idea and discovered the fact that the Iris pattern differed for each person. In 1987, they asked John Daugman to try creating actual algorithms to identify the iris. These algorithms obtained from Daugman in 1994 is the basis of all existing iris recognition systems and products (Daugman, 1993) and (Prasad et al., 2018).

\section{The Application}

Extensive applications for the iris system include access control to secure areas (buildings), control of distributed systems, secure financial transactions, credit card authentication, secure access to bank accounts, computer access or the database and counterterrorism. Iris systems are deployed in many countries for airline crews, airport staff, national ID cards, identification of missing children, the voting system in parliamentary and assembly polls and many others.

\section{Iris Recognition System}

Two operation modes most biometric systems are doing. Templates are added to a database by enrollment mode and an identification mode, where a template is created for an individual and then a match is found in the database of pre-registered templates.

The primary stages of an iris recognition system design include the following:

- Localization of pupil and iris

- Segmentation borders of the iris and the pupil

- Normalization of the iris part

- Feature extractions and

- Matching

Authentication is achieved by comparing the generated template to the iris image with the values templates which are stored in the database.

The matching is perform among one to many templates for the identification or the matching between one to one templates for verification.

\section{Related Work}

Jain et al. (2012) presented a biometric algorithm for iris recognition using Fast Fourier Transform and calculating all possible sets of Normalized Moment which are invariant to rotation and scale transformation. The Fast Fourier Transform converts image from spatial domain to frequency domain. It also filters noise in the image and gives more information that is precise. The paper used the CASIA iris image database ver. 1.0 and ver. 2.0. As a conclusion, the algorithm achieved a higher Correct Recognition Rate (Jain et al., 2012).

Mabrukar et al. (2013) presented a feature extraction method based on extracting the statistical features in an iris by binarizing the first and second order multi-scale Taylor coefficients using CASIA database on MATLAB. In their experiments, multi-scale Taylor-based features have pretty much immune to illumination changes. This is partially due to neglecting the 0th Taylor coefficient. Feature extraction using Multi-scale Taylor expansion was also implemented and it yielded good results (Mabrukar et al., 2013). Hosaini et al. (2013) compared the performance of Legendre moments, Zernike moments and Pseudo-Zernike moments in feature extraction for iris recognition. They have increased the moment orders until the best recognition rate was chieved. Robustness of these moments in various orders was evaluated in presence of White Gaussian Noise. Numerical results indicate that recognition rate by the Legendre; Zernike and Pseudo-Zernike moments in higher orders are approximately identical. However, average computation time for feature extraction is 4.5 , 18 and 0.75 seconds respectively for the Legendre, Zernike and Pseudo- Zernike moments of order 14. On the other hand, the result indicates that the Legendre moment is more robust than the others against the white Gaussian noise (Sarmah and Kumar, 2013). 
Sarmah and Kumar (2013) presented an algorithm based on Legendre moment. This algorithm takes advantage of the translation invariant property of the Legendre moments. So, it can reduce the computational cost for iris recognition matching on a larger iris image database. The system performed with a test on UPOL image database (Sarmah and Kumar, 2013).

Kaur et al. (2018) proposed a discrete orthogonal moment-based feature extraction that extracts global as well as local features. Krawtchouk moments extract local features; Tchebichef moments extract global characteristics of the entire image block. Dual-Hahn moments extract both global and local features, but the performance of the proposed method is evaluated on four publicly available databases achieving an improved accuracy of $99.80 \%$ for CASIAIrisV4- Interval, 99.90\% for IITD.v1, $100 \%$ for UPOL and $97.50 \%$ for UBIRIS.v2 as compared to the recently proposed methods. The technique was found to be robust for NIR as well as visible images under uncontrolled environmental conditions (Kaur et al., 2018).

Al-Juburi et al. (2017) presented a new iris recognition system using hybrid methods. These methods were used to extract features of tested eye images. Gabor wavelet and Zernike moment were used to extract features of iris. The proposed system was tested on CASIA-v4.0 interval database. The results show that the proposed method has a good accuracy about 97\%. PSNR is applied on the training and testing iris image to measure the simmilarity between them Al-Juburi et al. (2017).

Gnana et al. (2018) proposed an architecture for iris recognition and validated it on the dataset of visible images obtained from the University of Warsaw. They have under took a comparative analysis using LBPH features and Zernike features. They infer red that the proposed approach performed better with the visible images (Gnana et al., 2018).

\section{Methodology}

One of the main ways for iris recognition is to construct features vectors corresponding to individual iris images and perform iris matching based on some distance measurements. The extraction of features is a fundamental problem in the recognition of iris-based features that performance is greatly influenced by many parameters in the process of feature extraction (e.g., spatial location, direction, central frequency). It may vary depending on the environmental factors to acquire Iris image. There are many techniques used for feature extracting and merge two or more of these methods may produce a good result.

In image recognition, the rotation, scaling and translation invariant properties of image moments have a high significance. Therefore, $\mathrm{Hu}$ presented the use of moments for image analysis and pattern recognition (Hu, 1962). Legendre moments are classical orthogonal moment which are one of widest and most commonly moments used in recognition and image analysis (Oujaoura et al., 2014).

\section{Iris Localization and Segmentation}

Iris boundary detection is an important stage in the iris recognition system. Firstly, remove light reflection inside pupil by adjusting image intensity values and filled the holes (Fig. 2a).

The next step is to find the pupil center and pupil radius by the Hough transform. In our case, give the approximate lowest and highest radius of the pupil as input (Fig. 2b).

Then compute iris radii to crop the iris region from the eye image (Fig. 2d).

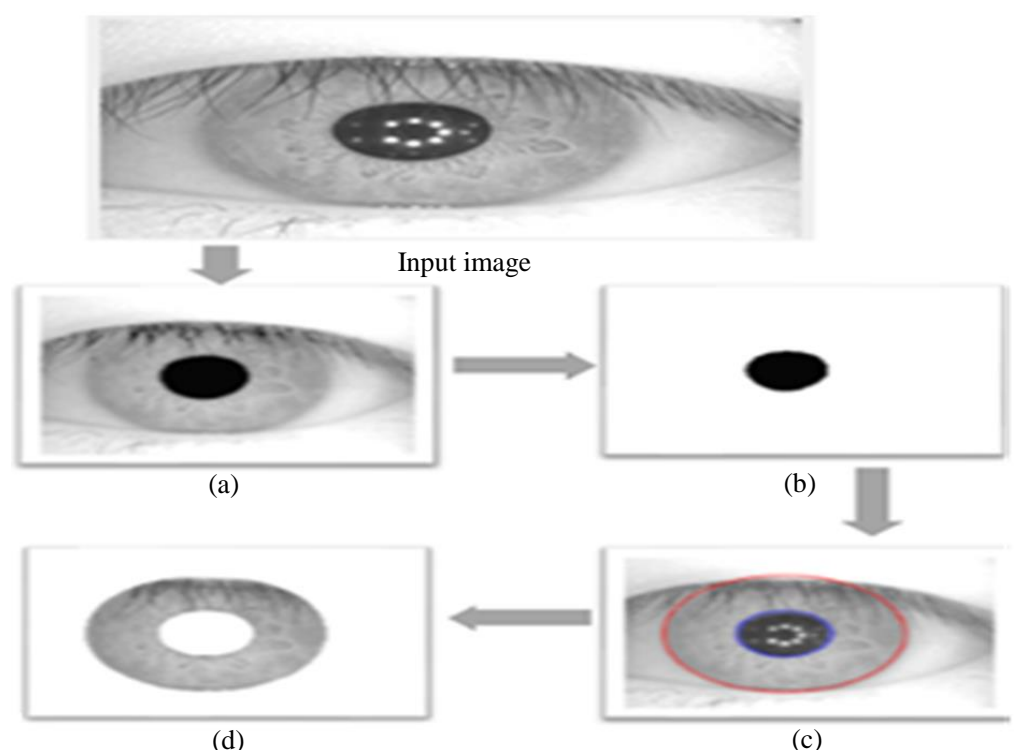

Fig. 2: Steps of localization and segmentation 


\section{Iris Normalization}

After computing the inner and outer circles of the iris, the iris region is segmented out and normalized by a convert from polar coordinate to the Cartesian coordinate for easy computations, as shown in (Fig. 3) (Daugman, 1993). The polar coordinates are defined by $r$ (the radial coordinate) and $\times \theta$ (the angular coordinate often called polar angle) while Cartesian coordinates are defined in $x$ and $y$ (Equation 1) to get the iris region as matric of data:

$$
x=r \cos \theta, y=r \sin \theta
$$

One of the problems in an iris recognition system is the occlusion that happens due to eyelashes and eyelids as shown in (Fig. 4). This occlusion increases the complexity and affects the performance of matching and feature extraction processes.

It was done by applying the proposed approach in many iris regions, in order to select a Region Of Interest
(ROI) from the iris area by avoiding the regions that occlusion may occur in.

In the following, the five regions were imposed for experimentation:

a) Upper region

b) Down region

c) Two sides region

d) The circular region around the pupil

e) The circular region around the pupil + two sides region, as shown in (Fig. 5)

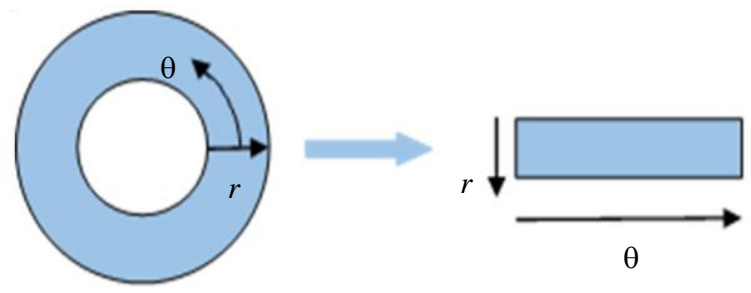

Fig. 3: Daugman's rubber sheet model to conversion from Polar to Cartesian

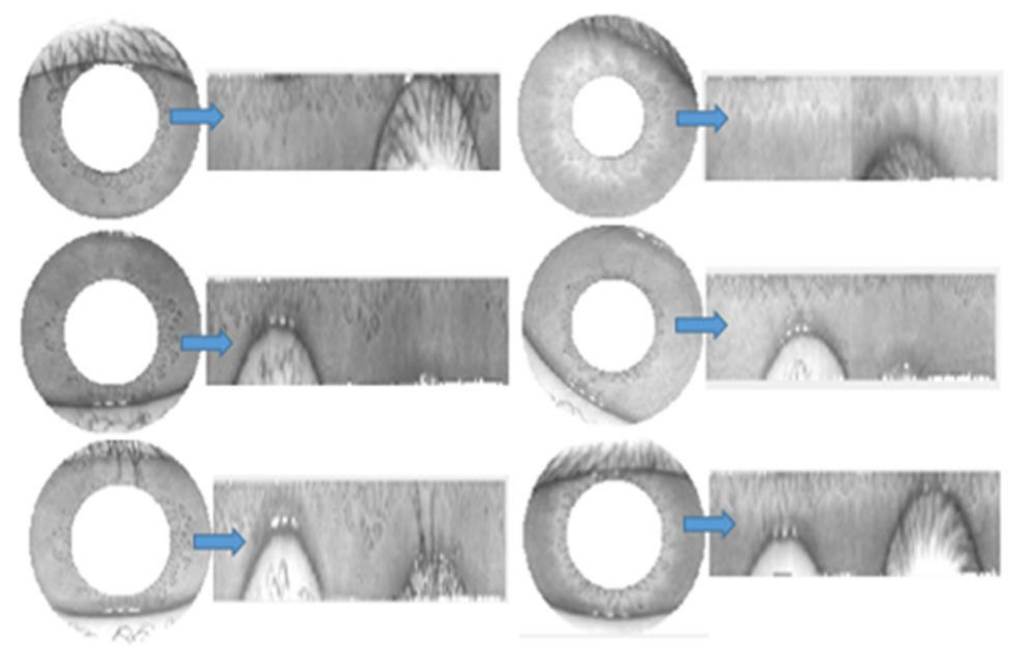

Fig. 4: Sample of occlusion that happens due to eyelids and eyelashes

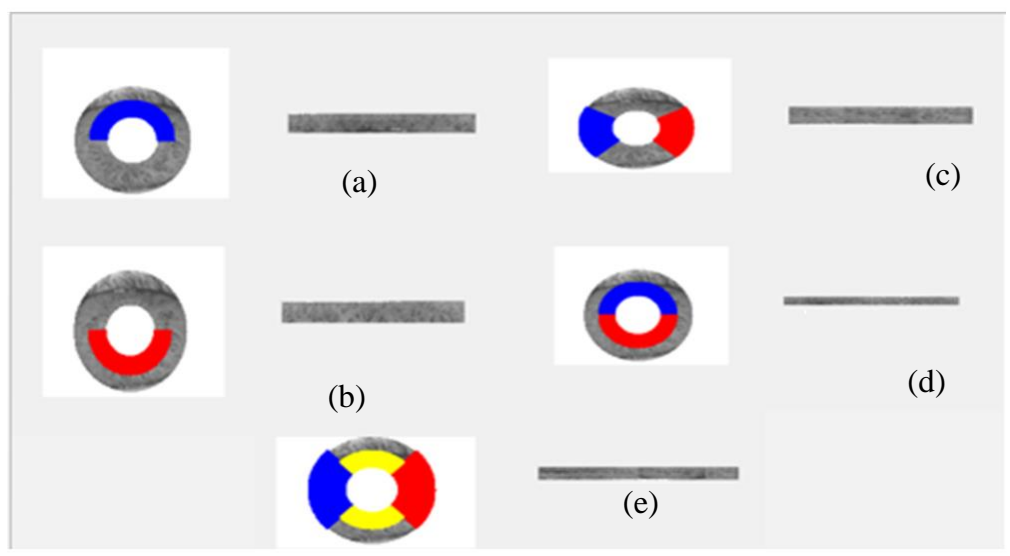

Fig. 5: Iris regions for experimentation 


\section{Features Extraction}

The extraction of features remains a significant phase in recognition system using iris. A successful recognition rate and reduction in recognition time of two iris templates mostly depend on efficient feature extraction technique. A great deal of information about the image at a higher level can be contained in small patterns of qualitative differences in the local gray level by using local pattern features such as Local Binary (LBP), Local Triangular (LTP) and Local Quadrant Pattern (LQP). Local patterns have proven very successful in visual recognition tasks ranging from texture classification to face analysis and object detection.

\section{A. Legendre Moments}

The two-dimensional Legendre moments of order $(p$, $q$ ) with image intensity function $f(x, y)$ are defined as:

$L_{p, q}=\frac{(2 p+1)(2 q+1)}{4} \iint_{-1}^{1} P_{p}(x) P_{q}(y) f(x, y) d x d y$,

where $p, q=0,1,2 \ldots \infty$

The kernel functions $P$ denote Legendre polynomials of order $p$ :

$P_{p}(x)=\sum_{k=0}^{p}\left\{\frac{(-1)^{\frac{p-k}{2} x^{k}(p+k) !}}{2^{p} k !\left(\frac{p-k}{2}\right) !\left(\frac{p+k}{2}\right) !}\right\}_{p-k=\text { even }}$

And, the recurrent formula of Legendre polynomials is:

$$
\left\{\begin{array}{l}
P_{p+1}(x)=\frac{2 p+1}{p+1} x P_{p}(x)-\frac{p}{p+1} P_{p-1}(x) \\
P_{1}(x)=x \\
P_{0}(x)=1
\end{array}\right.
$$

To compute Legendre moments from a digital image, the integrals in previous (Equation 2) are replaced by summations and the coordinates of the image must be normalized into $[-1 ; 1]$.

Therefore, the numerical approximate form of Legendre moments, for a discrete image of $N \times M$ pixels with intensity's function $f(x ; y)$ is:

$$
L_{p q}=\frac{(2 p+1)(2 q+1)}{M N} \sum_{i=0}^{M-1} \sum_{j=0}^{N-1} P_{p}\left(x_{i}\right) P_{q}\left(y_{j}\right) f\left(x_{i}, y_{j}\right)
$$

where, $x_{i}$ and $y_{j}$ denote the normalized pixel coordinates in the range of $[-1 ; 1]$, which are given by:

$$
x_{i}=\frac{2 i-(M-1)}{M-1}, y_{j}=\frac{2_{j}-(N-1)}{N-1}
$$

Symmetry and recursion properties of the orthogonal basis function can be exploited to speed up the computation (Oujaoura et al., 2014).

\section{B. Local Quadrant Pattern (LQP)}

Hussain and Trigges suggested LQP operator as development for LBP of visual recognition. The LBP method extracts a binary descriptor by creating intensity of the central pixel as the threshold of his neighborhood for each pixel of an image (ul Hussain and Triggs, 2012). Figure 6 gives an illustration with an example of eight neighbors equally spaced around the central pixel.

Let $I c$ and $I p(p=1,2, \ldots 8)$ denote the intensity of the central pixel and its neighbors, respectively.

The operator is performed by the binary test as follows (Equation 7):

$$
L B P_{R, N}=\sum_{p=0}^{N-1} f\left(I_{p}-I_{c}\right) 2^{p}, f(x)=\left\{\begin{array}{l}
1 x \geq 0 \\
0<0
\end{array}\right.
$$

where, $R$ denotes the different sampling radius and $N$ represents the number of the sample points equally spaced around the circle. A binary code with $N$ bits is obtained from each pixel. So will results in $2^{N}$ different patterns. Finally, we convert these patterns to a decimal value.

The LQP collects the directional geometric features in Horizontal (H), Vertical (V), Diagonal (D) and Antdiagonal (A) strips of pixels and combinations of these (HVDA) (Fig. 7a).

Local Quadrant Pattern is a new method proposed that is based on the idea of LTP (Al-Jawahry and Mohammed, 2019).

First, the difference between the center pixel (IC) and each neighbor pixels ( $I i)$ as (Equation 8) is calculated:

$D i=I i-I c ; i=1,2, \ldots 8$

After that, every two results $D_{i}$ for a specific direction will be put in one vector accordant (Equation 9) (Rao and Rao, 2015) as shown in (Fig. 7b):

$$
p_{\alpha}=F\left(D_{i}, D_{i+45}\right) ; i=(1+\alpha / 45), \forall \alpha=0^{\circ}, 45^{\circ}, 90^{\circ}, 135^{\circ}
$$

hen, (Equation 10) is applied on each resulted value $p_{\alpha}$ from (Equation 9):

$F_{i}\left\{\begin{array}{l}3\left|p_{i 1}\right|>t \cap\left|p_{i 2}\right|>t \cap \operatorname{sign}\left(p_{i 1}\right) \neq \operatorname{sign}\left(p_{i 2}\right) \\ 2\left|p_{i 1}\right|>t \cap\left|p_{i 2}\right|>t \cap \operatorname{sign}\left(p_{i 1}\right) \neq \operatorname{sign}\left(p_{i 2}\right) \\ 1 \quad\left|p_{i 1}\right| \leq t \cap\left|p_{i 2}\right| \geq t \cup\left|p_{i 1}\right| \geq t \cap\left|p_{i 2}\right| \leq t \\ 0 \quad \text { else, }\end{array}\right.$

Where:

$F_{i}=$ Result each line, $i=1,2, \ldots 4$

$t=$ Specific threshold 


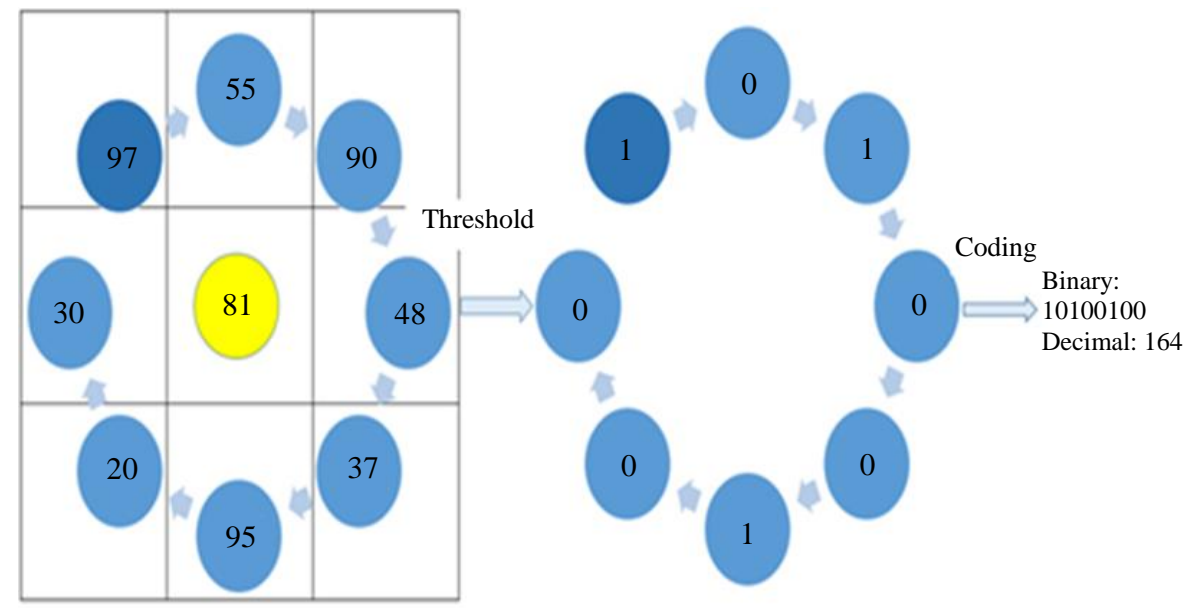

Fig. 6: A conventional LBP coding
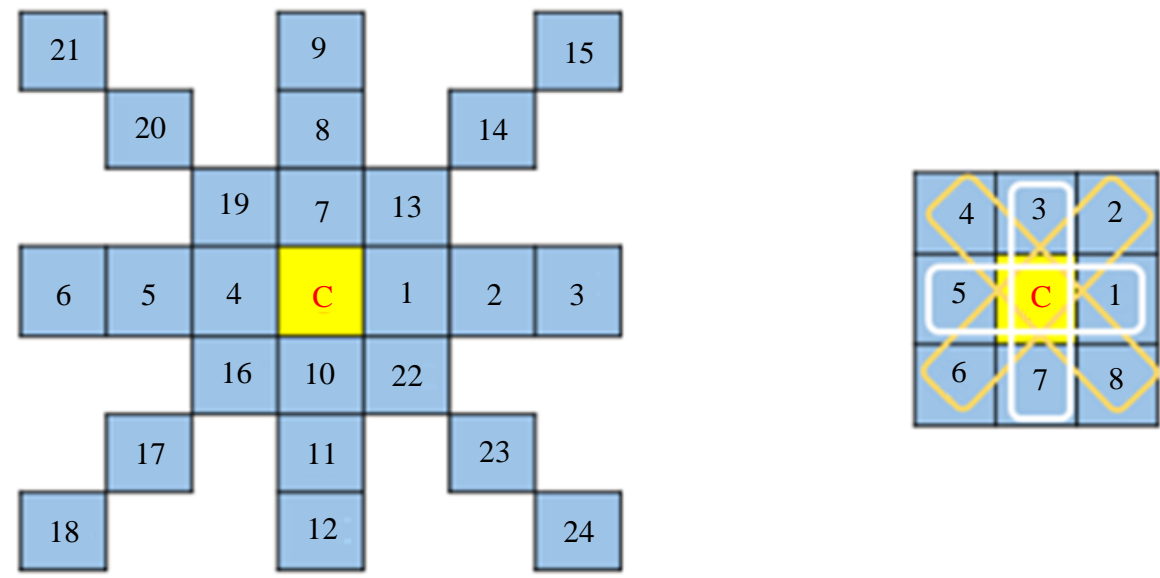

(a)

(b)

Fig. 7: The LQP calculate for given $7 \times 7$ pattern using HVDA geometric structure (a) for given $3 \times 3$ pattern

Finally, these values are converted to decimal number and summation to get a new value of center pixel LQP as follows (Equation 11):

$$
L Q \mathrm{P}_{\text {row }}=\sum_{i=0}^{\text {row }-1} F_{i} \times 2^{i}
$$

where, $F_{i}$ value results from (Equation 10).

The feature vector consists of two parts: first, $V_{1}$ computes Legendre moment for $f(x, y)$ matric resulted from iris normalization stage. Second, $V_{2}$ is resulted from applying LQP method to $f(x, y)$; then computing Legendre moment; and finally generating $V_{3}$ appended $V_{1}$ by $V_{2}$.

\section{Matching}

For matching, City Block Distance is used due to get a higher recognition accuracy ratio than other methods (Sari et al., 2018). City Block Distance calculates the absolute difference between two vectors according to (Equation 12). In the proposed system the features vector is got from previously mentioned techniques:

$$
d(Q, V)=\sum_{i=1}^{N}\left|Q_{i}-V_{i}\right|
$$

\section{Results and Discussion}

The proposed approach is implemented and tested on CASIA-V4-Interval database. The developed system was established using MATLAB (version R2017a) programming language. The programs work under Windows 10 operating system, laptop Computing time calculate Average Recognition Time (ART) at second. Table 13 shows computed ART by calculating the average time for comparison of each testing image with all training images from the database.

In Tables 1 to 5 for left eye images and in Tables 6 to 10 for right eye images, showed that increasing of Legendre order lead to increase of recognition rate, in this case just Legendre be used for extraction feature of image, these steps are shown in (Fig. 8 part C1). As shown in Fig 9 and 10, the system utilized LQP followed by Legendre, this approach resulted high recognition rate, 


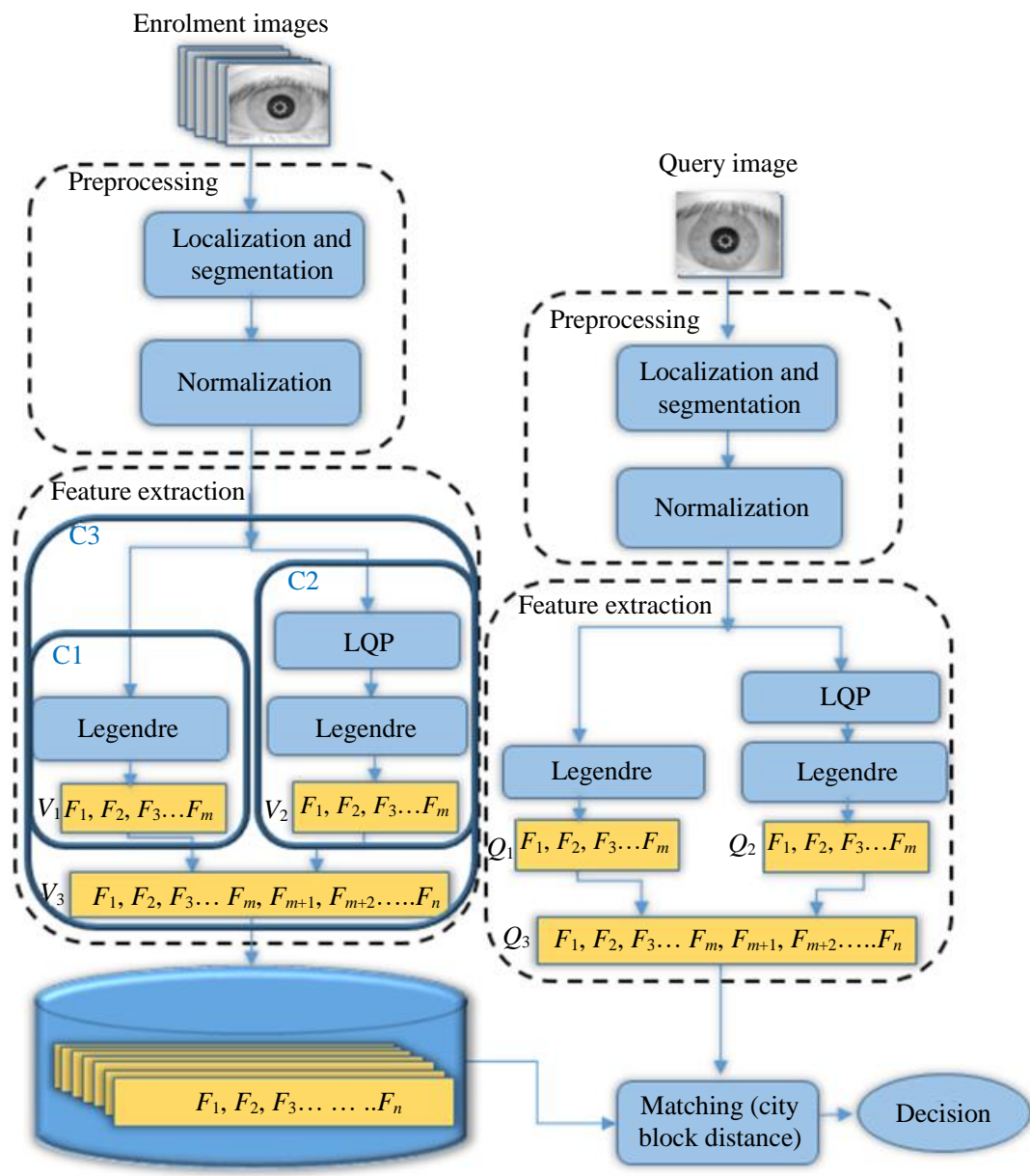

Fig. 8: Block diagram of proposed iris recognition system

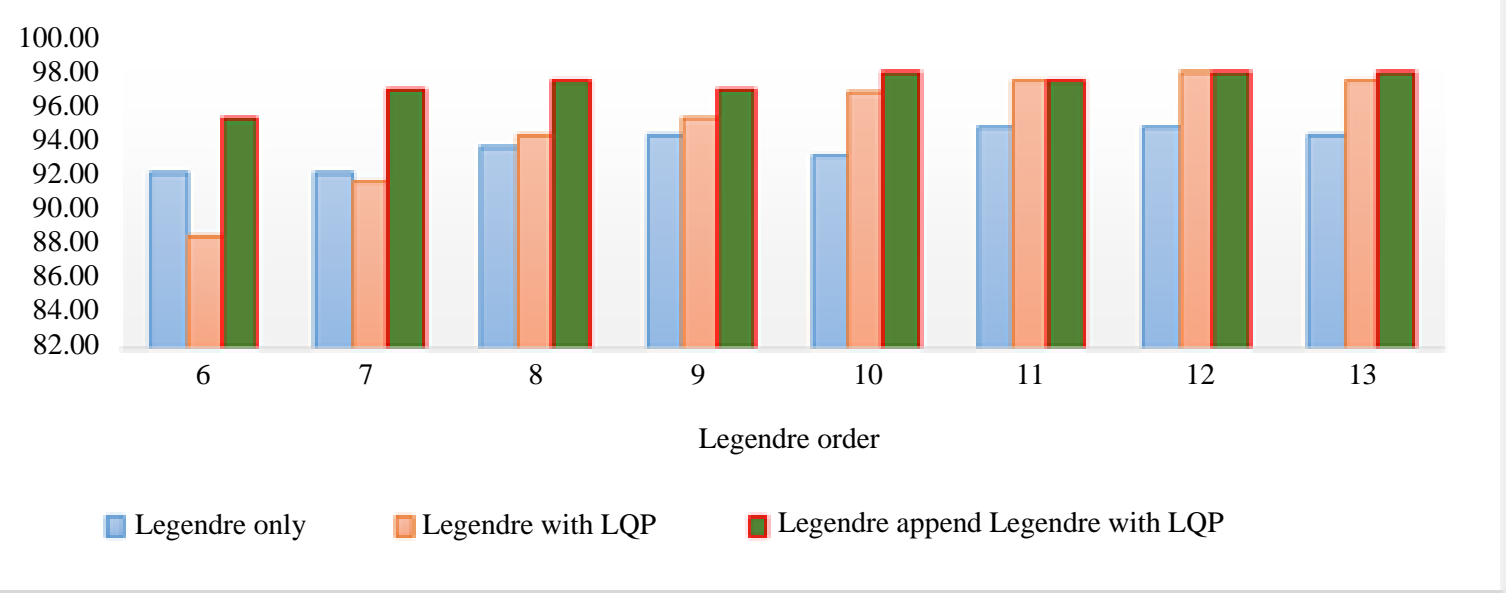

Fig. 9: Accuracy ratio for left eye (down iris region) using Legendre moment only, Legendre with LQP and Legendre appended Legendre with LQP (5 enroll: 5 test) 


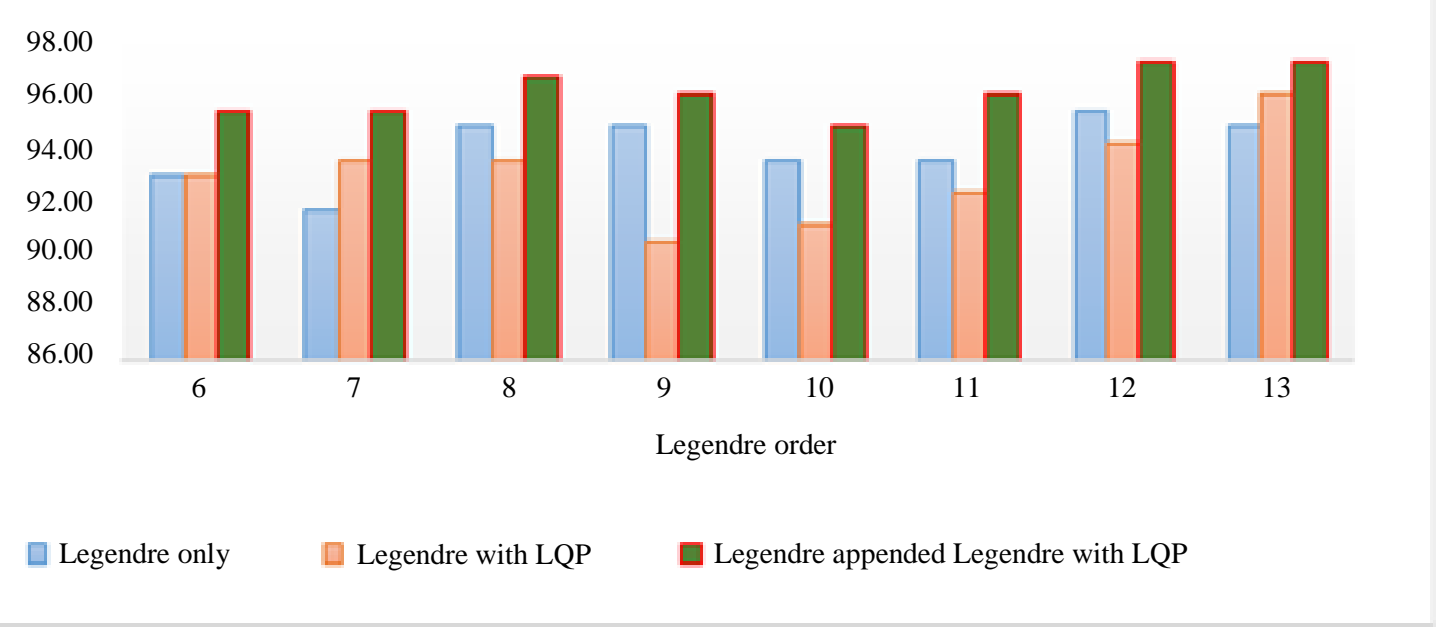

Fig. 10: Accuracy ratio for right eye (down iris region) using Legendre moment only, Legendre with LQP and Legendre appended Legendre with LQP (5 enroll: 5 test)

Table 1: Recognition accuracy ratio when enrolment set is changed and testing set for left eye image (upper iris region) using Legendre moment only

\begin{tabular}{llllll}
\hline Legendre order & 5 enroll: 5 test & 6 enroll: 4 test & 7 enroll: 3 test & 8 enroll: 2 test & 9 enroll: 1 test \\
\hline 6th & 88.42 & 91.45 & 88.60 & 97.37 & 94.74 \\
7th & 90.53 & 92.11 & 89.47 & 97.37 & 94.74 \\
8th & 91.05 & 91.45 & 91.23 & 96.05 & 94.74 \\
9th & 91.58 & 93.42 & 91.23 & 96.05 & 94.74 \\
10th & 92.63 & 92.76 & 90.35 & 94.74 & 94.74 \\
11th & 91.58 & 91.45 & 90.35 & 96.05 & 94.74 \\
12th & 92.63 & 93.42 & 91.23 & 96.05 & 94.74 \\
13th & 92.11 & 93.42 & 89.47 & 94.74 & 94.74 \\
\hline
\end{tabular}

Table 2: Recognition accuracy ratio when enrolment set is changed and testing set for left eye image (down iris region) using Legendre moment only

\begin{tabular}{llllll}
\hline Legendre order & 5 enroll: 5 test & 6 enroll: 4 test & 7 enroll: 3 test & 8 enroll: 2 test & 9 enroll: 1 test \\
\hline 6th & 92.11 & 91.45 & 94.74 & 96.05 & 100.00 \\
7th & 92.11 & 90.79 & 95.61 & 96.05 & 100.00 \\
8th & 93.68 & 92.11 & 96.49 & 96.05 & 100.00 \\
9th & 94.21 & 92.11 & 96.49 & 96.05 & 100.00 \\
10th & 93.16 & 91.45 & 96.49 & 96.05 & 100.00 \\
11th & 94.74 & 92.11 & 96.49 & 96.05 & 100.00 \\
12th & 94.74 & 92.76 & 95.61 & 96.05 & 100.00 \\
13th & 94.21 & 92.76 & 94.74 & 94.74 & 100.00 \\
\hline
\end{tabular}

Table 3: Recognition accuracy ratio when enrolment set is changed and testing set for left eye image (two sides iris region) using Legendre moment only

\begin{tabular}{llllll}
\hline Legendre order & 5 enroll: 5 test & 6 enroll: 4 test & 7 enroll: 3 test & 8 enroll: 2 test & 9 enroll: 1 test \\
\hline 6th & 84.74 & 87.50 & 92.11 & 92.11 & 94.74 \\
7th & 88.95 & 91.45 & 94.74 & 93.42 & 94.74 \\
8th & 89.47 & 91.45 & 94.74 & 93.42 & 94.74 \\
9th & 92.63 & 95.39 & 95.61 & 93.42 & 94.74 \\
10th & 92.63 & 95.39 & 95.61 & 93.42 & 94.74 \\
11th & 92.11 & 93.42 & 96.49 & 94.74 & 94.74 \\
12th & 92.11 & 96.05 & 96.49 & 94.74 & 94.74 \\
13th & 92.63 & 94.74 & 94.74 & 94.74 & 94.74 \\
\hline
\end{tabular}


Table 4: Recognition accuracy ratio when enrolment set is changed and testing set for left eye image (the circular region around the pupil) using Legendre moment only

\begin{tabular}{llllll}
\hline Legendre order & 5 enroll: 5 test & 6 enroll: 4 test & 7 enroll: 3 test & 8 enroll: 2 test & 9 enroll: 1 test \\
\hline 6th & 81.58 & 82.89 & 86.84 & 86.84 & 86.84 \\
7th & 84.74 & 86.18 & 88.60 & 89.47 & 92.11 \\
8th & 85.26 & 87.50 & 87.72 & 90.79 & 92.11 \\
9th & 87.37 & 88.16 & 88.60 & 92.11 & 94.74 \\
10th & 88.95 & 91.45 & 93.86 & 93.42 & 92.11 \\
11th & 90.00 & 90.79 & 91.23 & 92.11 & 94.74 \\
12th & 90.00 & 92.11 & 92.98 & 92.11 & 94.74 \\
13th & 91.05 & 92.11 & 92.11 & 90.79 & 94.74 \\
\hline
\end{tabular}

Table 5: Recognition accuracy ratio when enrolment set is changed and testing set for left eye image (the circular region around the pupil + Sides region) using Legendre moment only

\begin{tabular}{llllll}
\hline Legendre order & 5 enroll: 5 test & 6 enroll: 4 test & 7 enroll: 3 test & 8 enroll: 2 test & 9 enroll: 1 test \\
\hline 6th & 78.42 & 79.61 & 82.46 & 84.21 & 94.74 \\
7th & 83.68 & 84.87 & 85.96 & 86.84 & 94.74 \\
8th & 84.21 & 85.53 & 88.60 & 89.47 & 94.74 \\
9th & 84.21 & 87.50 & 91.23 & 92.11 & 94.74 \\
10th & 84.74 & 86.84 & 90.35 & 90.79 & 92.11 \\
11th & 84.21 & 88.16 & 88.60 & 88.16 & 92.11 \\
12th & 84.21 & 87.50 & 87.72 & 86.84 & 92.11 \\
13th & 84.21 & 87.50 & 88.60 & 88.16 & 89.47 \\
\hline
\end{tabular}

Table 6: Recognition accuracy ratio when enrolment set is changed and testing set for right eye image (upper iris region) using Legendre moment only

\begin{tabular}{llllll}
\hline Legendre order & 5 enroll: 5 test & 6 enroll: 4 test & 7 enroll: 3 test & 8 enroll: 2 test & 9 enroll: 1 test \\
\hline 6th & 85.00 & 84.72 & 88.89 & 91.67 & 94.44 \\
7th & 88.89 & 88.89 & 90.74 & 91.67 & 88.89 \\
8th & 90.56 & 90.97 & 90.74 & 93.06 & 94.44 \\
9th & 92.22 & 92.36 & 91.67 & 94.44 & 94.44 \\
10th & 92.22 & 92.36 & 92.59 & 93.06 & 91.67 \\
11th & 93.89 & 93.75 & 93.52 & 95.83 & 97.22 \\
12th & 92.78 & 93.06 & 93.52 & 94.44 & 94.44 \\
13th & 93.89 & 95.14 & 94.44 & 94.44 & 94.44 \\
\hline
\end{tabular}

Table 7: Recognition accuracy ratio when enrolment set is changed and testing set for right eye image (down iris region) using Legendre moment only

\begin{tabular}{llllll}
\hline Legendre order & 5 enroll: 5 test & 6 enroll: 4 test & 7 enroll: 3 test & 8 enroll: 2 test & 9 enroll: 1 test \\
\hline 6th & 92.22 & 93.06 & 91.67 & 91.67 & 100.00 \\
7th & 91.11 & 90.28 & 90.74 & 93.06 & 97.22 \\
8th & 93.89 & 94.44 & 93.52 & 94.44 & 97.22 \\
9th & 93.89 & 94.44 & 93.52 & 94.44 & 94.44 \\
10 th & 92.78 & 92.36 & 90.74 & 91.67 & 94.44 \\
11 th & 92.78 & 93.06 & 91.67 & 91.67 & 97.22 \\
12th & 92.78 & 93.06 & 91.67 & 91.67 & 97.22 \\
13th & 93.89 & 95.14 & 94.44 & 95.83 & 97.22 \\
\hline
\end{tabular}

Table 8: Recognition accuracy ratio when enrolment set is changed and testing set for right eye image (sides iris region) using Legendre moment only

\begin{tabular}{llllll}
\hline Legendre order & 5 enroll: 5 test & 6 enroll: 4 test & 7 enroll: 3 test & 8 enroll: 2 test & 9 enroll: 1 test \\
\hline 6th & 88.89 & 88.19 & 94.44 & 94.44 & 94.44 \\
7th & 90.56 & 89.58 & 94.44 & 94.44 & 94.44 \\
8th & 90.56 & 89.58 & 94.44 & 97.22 & 97.22 \\
9th & 90.00 & 89.58 & 94.44 & 95.83 & 94.44 \\
10th & 91.11 & 90.28 & 92.59 & 93.06 & 97.22 \\
11th & 92.22 & 91.67 & 94.44 & 93.06 & 94.44 \\
12th & 92.22 & 91.67 & 95.37 & 94.44 & 97.22 \\
13th & 92.78 & 91.67 & 94.44 & 93.06 & 94.44 \\
\hline
\end{tabular}


Table 9: Recognition accuracy ratio when enrolment set is changed and testing set for right eye image (the circular region around the pupil) using Legendre moment only

\begin{tabular}{llllll}
\hline Legendre order & 5 enroll: 5 test & 6 enroll: 4 test & 7 enroll: 3 test & 8 enroll: 2 test & 9 enroll: 1 test \\
\hline 6th & 80.56 & 79.17 & 81.48 & 84.72 & 88.89 \\
7th & 82.78 & 81.94 & 85.19 & 90.28 & 94.44 \\
8th & 85.56 & 85.42 & 88.89 & 93.06 & 97.22 \\
9th & 87.22 & 86.81 & 87.96 & 90.28 & 94.44 \\
10th & 88.89 & 89.58 & 88.89 & 93.06 & 100.00 \\
11th & 88.33 & 89.58 & 90.74 & 93.06 & 100.00 \\
12th & 89.44 & 89.58 & 89.81 & 91.67 & 100.00 \\
13th & 89.44 & 89.58 & 89.81 & 90.28 & 97.22 \\
\hline
\end{tabular}

Table 10: Recognition accuracy ratio when enrolment set is changed and testing set for right eye image ( the circular region around the pupil + Sides region ) using Legendre moment only

\begin{tabular}{llllll}
\hline Legendre order & 5 enroll: 5 test & 6 enroll: 4 test & 7 enroll: 3 test & 8 enroll: 2 test & 9 enroll: 1 test \\
\hline 6th & 81.67 & 83.33 & 85.19 & 86.11 & 88.89 \\
7th & 83.33 & 85.42 & 89.81 & 90.28 & 94.44 \\
8th & 85.00 & 86.11 & 88.89 & 91.67 & 94.44 \\
9th & 86.11 & 86.81 & 87.96 & 91.67 & 91.67 \\
10th & 86.11 & 88.19 & 88.89 & 91.67 & 91.67 \\
11th & 86.11 & 86.11 & 87.96 & 90.28 & 88.89 \\
12th & 87.22 & 86.81 & 87.04 & 90.28 & 91.67 \\
13th & 86.67 & 86.11 & 87.04 & 90.28 & 88.89 \\
\hline
\end{tabular}

Table 11: Recognition accuracy ratio when enrolment set is changed and testing set for left eye image (best ROI (down)) using Legendre moment only appended by Legendre moment with LQP.

\begin{tabular}{llllll}
\hline Legendre order & 5 enroll: 5 test & 6 enroll: 4 test & 7 enroll: 3 test & 8 enroll: 2 test & 9 enroll: 1 test \\
\hline 6th & 95.26 & 95.39 & 99.12 & 97.37 & 100.00 \\
7th & 96.84 & 96.71 & 98.25 & 97.37 & 100.00 \\
8th & 97.37 & 96.71 & 98.25 & 97.37 & 100.00 \\
9th & 96.84 & 96.05 & 98.25 & 97.37 & 100.00 \\
10th & $\mathbf{9 7 . 8 9}$ & 98.03 & 98.25 & 97.37 & 100.00 \\
11th & 97.37 & 97.37 & 98.25 & 97.37 & 100.00 \\
12th & 97.89 & 97.37 & 98.25 & 97.37 & 100.00 \\
13th & 97.89 & 97.37 & 98.25 & 97.37 & 100.00 \\
\hline
\end{tabular}

Table 12: Recognition accuracy ratio when change enrolment set and testing set for right eye image (best ROI (two sides)) using Legendre moment only appended by Legendre moment with LQP.

\begin{tabular}{llllll}
\hline Legendre order & 5 enroll: 5 test & 6 enroll: 4 test & 7 enroll: 3 test & 8 enroll: 2 test & 9 enroll: 1 test \\
\hline 6th & 94.44 & 94.44 & 97.22 & 98.61 & 97.22 \\
7th & 96.11 & 94.44 & 97.22 & 97.22 & 97.22 \\
8th & 96.11 & 95.14 & 97.22 & 97.22 & 97.22 \\
9th & 96.67 & 95.14 & 97.22 & 97.22 & 97.22 \\
10th & 97.22 & 96.53 & 97.22 & 97.22 & 97.22 \\
11th & 96.67 & 95.83 & 97.22 & 97.22 & 97.22 \\
12th & 97.22 & 96.53 & 98.15 & 98.61 & 100.00 \\
13th & 97.22 & 96.53 & 98.15 & 98.61 & 100.00 \\
\hline
\end{tabular}

Table 13: Explain features count for each Legendre order and average recognition time (ART) at second using Legendre moment only and Legendre appended Legendre with LQP for (best ROI (down))

\begin{tabular}{|c|c|c|c|c|c|c|}
\hline \multirow[b]{2}{*}{ Legendre order } & \multicolumn{3}{|c|}{ ART Legendre only } & \multicolumn{3}{|c|}{ ART for Legendre with LQP } \\
\hline & Feature count & Left eye & Right eye & Feature count & Left eye & Right eye \\
\hline 6th & 25 & 0.65 & 0.69 & 50 & 0.81 & 0.80 \\
\hline 7 th & 33 & 0.75 & 0.73 & 66 & 0.97 & 0.96 \\
\hline 8th & 43 & 0.90 & 0.87 & 84 & 1.23 & 1.23 \\
\hline 9th & 52 & 1.12 & 1.10 & 104 & 1.69 & 1.70 \\
\hline 10th & 63 & 1.49 & 1.47 & 126 & 2.36 & 2.37 \\
\hline 11 th & 75 & 2.10 & 2.08 & 150 & 3.72 & 3.50 \\
\hline 12 th & 88 & 3.09 & 2.97 & 176 & 5.43 & 5.48 \\
\hline 13th & 102 & 4.54 & 4.65 & 204 & 8.48 & 8.39 \\
\hline
\end{tabular}


As shown with Tables 11 and 12 there three steps for feature extractor, first step the image enrolled to Legendre algorithm to produce vector of features (named $\mathrm{V}_{1}$ ). Second one, the image enrolled to LQP algorithm to produce array of coefficients that enrolled as inputs to the Legendre to produce vector of features $\left(\mathrm{V}_{2}\right)$. Finally, fusion stages has been applied by append vector of $\mathrm{V}_{1}$ to $\mathrm{V}_{2}$ to produce the final vector of all features (named $V_{3}$ ). Best results with high recognition rate have been satisfied based on this fusion.

\section{Conclusion and Future Work}

- The best order for Legendre is 10th, where it gives high recognition rate as well as a resistance to variation of images

- The best result, have gotten with down part of left eye while the high recognition rate has been satisfied with two sides of right eye as shown with Fig 11 and 12

- Fusion of features of Legendre and LQP gave the best results with minima errors

- In the future, we are going to apply the proposed approach with other databases

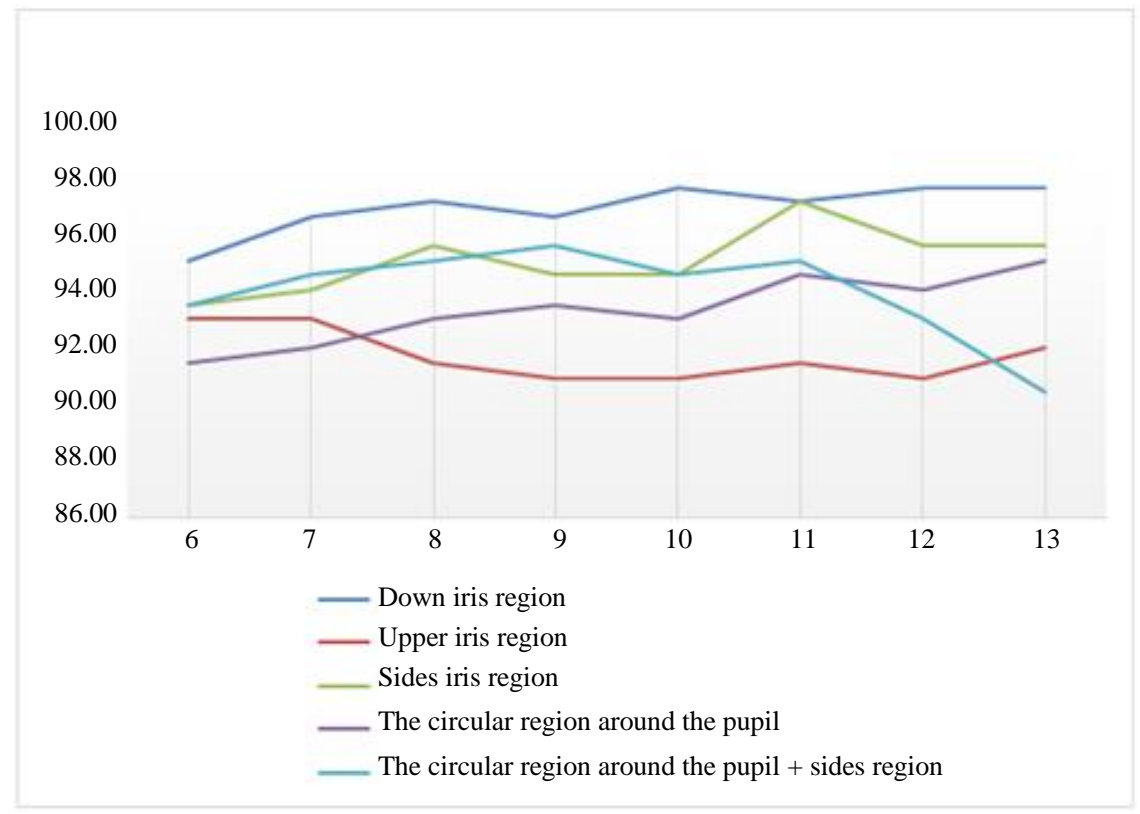

Fig. 11: Accuracy ratio for left eye to different regoins using Legendre only appended Legendre with LQP (5 enroll: 5 test)

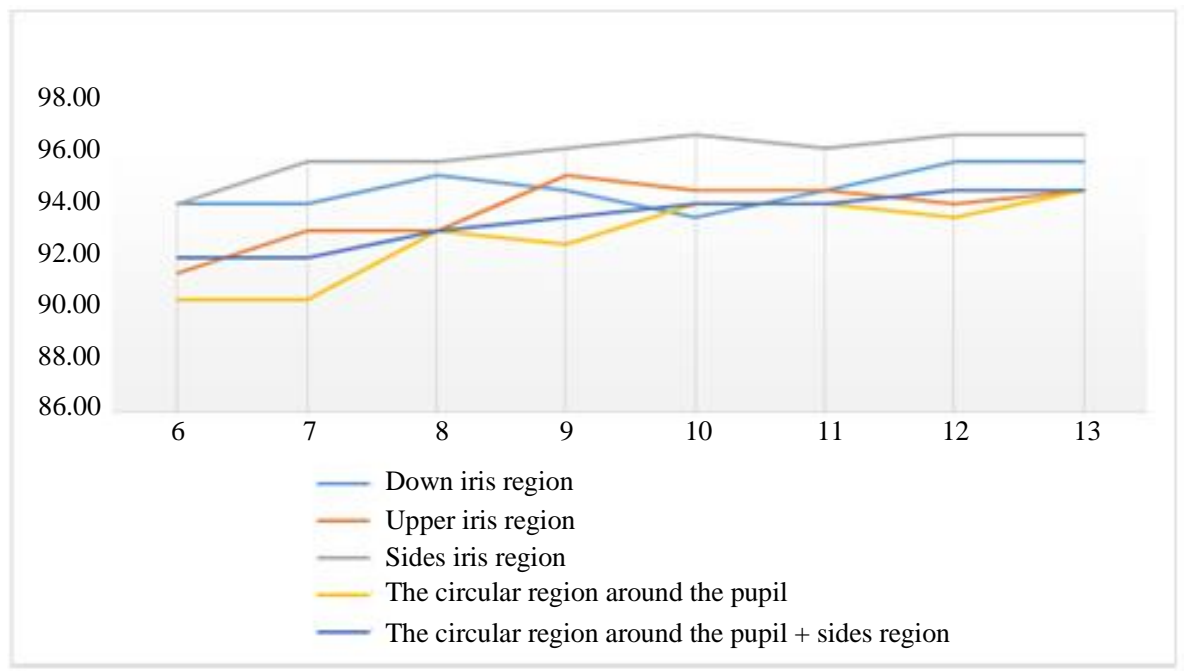

Fig. 12: Accuracy ratio for right eye to different regoins using Legendre only appended Legendre with LQP (5 enroll: 5 test) 


\section{Author's Contributions}

Asaad Noori Hashim: Has contributed to the analysis and simulated of the proposed algorithm. Also, he contributed to the write-up and language revision.

Bushraa Mahdi Al-Hashimi: Has contributed to review the literature, identified the research gap and revising the results.

\section{Ethics}

This paper is genuine and includes unpublished material. The corresponding author confirms that the coauthor has read and approved the manuscript and no ethical issues involved.

\section{References}

Al-Jawahry, H.M. and H.R. Mohammed, 2019. Local Quadrant Pattern with Co-occurrence Matrix (LQPCM): Hybrid method for image classification and feature extraction. J. Eng. Applied Sci., 14: 2171-2176. DOI: 10.3923/jeasci.2019.2171.2176

Al-Juburi, B.J.A., H.R. Mohammed and A.N.H. AlShareefi, 2017. Iris recognitions identification and verification using hybrid techniques. Res. J. Applied Sci. Eng. Technol., 14: 473-482.

DOI: $10.19026 /$ rjaset.14.5150

Daugman, J.G., 1993. High confidence visual recognition of persons by a test of statistical independence. IEEE Tran. Pattern Anal. Mach. Intell., 15: 1148-1161. DOI: 10.1109/34.244676

Gnana, P.R., V.M. Ravi and K.M. Sriraam, 2018. Iris recognition using visible images based on the fusion of daugman's approach and Hough transform. Proceedings of the 2nd International Conference on Biometric Engineering and Applications, May 16-18, ACM, pp: 24-29. DOI: 10.1145/3230820.3230825

Hosaini, S.J., S. Alirezaee, M. Ahmadi and S.V.A.D. Makki, 2013. Comparison of the Legendre, Zernike and Pseudo-Zernike moments for feature extraction in iris recognition. Proceedings of the 5th International Conference and Computational Intelligence and Communication Networks, Sept. 27-29, IEEE Xplore Press, Mathura, India, pp: 225-228.

DOI: $10.1109 /$ CICN.2013.54
Hu, M.K., 1962. Visual pattern recognition by moment invariants. IRE Trans. Inform. Theory, 8: 179-87. DOI: $10.1109 /$ TIT.1962.1057692

Jain, B., M.K. Gupta and J. Bharti, 2012. Efficient iris recognition algorithm using method of moments. Int. J. Artificial Intell. Applic.

Kaur, B., S. Singh and J. Kumar, 2018. Robust iris recognition using moment invariants. Wireless Personal Commun., 99: 799-828. DOI: $10.1007 / \mathrm{s} 11277-017-5153-8$

Mabrukar, S.S., N.S. Sonawane and J.A. Bagban, 2013. Biometric system using Iris pattern recognition. Int. J. Innovat. Technol. Explor. Eng., 2: 54-57.

Oujaoura, M., B. Minaoui and M. Fakir, 2014. Image annotation by moments. Moments Moment Invariants-Theory Applic., 1: 227-252. DOI: $10.15579 /$ gcsr.vol1.ch10, GCSR Vol

Prasad, M.R., T.C. Manjunath, M.D.A. Bhyratae and N. Kumar, 2018. Design and development of IRIS biometric systems-a exhaustive review summary and problem formulation. Int. J. Res., 7: 1-14.

Rao, L.K. and D.V. Rao, 2015. Local quantized extrema patterns for content-based natural and texture image retrieval. Human-Centric Comput. Inform. Sci., 5: 26-26. DOI 10.1186/s13673-015-0044-z

Sari, Y., M. Alkaff and R.A. Pramunendar, 2018. Iris recognition based on distance similarity and PCA. Proceedings of the 4th International Conference on Engineering, Technology and Industrial Application, (TIA' 18), 020044-020044. DOI: $10.1063 / 1.5042900$

Sarmah, A. and C.J. Kumar, 2013. Iris verification using Legendre moments and KNN classifier. Int. J. Eng. Sci. Invent., 2: 52-59.

ul Hussain, S. and B. Triggs, 2012. Visual recognition using local quantized patterns. Proceedings of the 12th European Conference on Computer Vision, Oct. 07-13, Springer-Verlag Berlin, pp: 716-729. 